\title{
Treatment of complications from polyacrylamide hydrogel breast augmentation
}

\author{
WANG WEI \\ Department of Plastic Surgery, The First People's Hospital of Xuzhou, Xuzhou, Jiangsu 221000, P.R. China
}

Received October 27, 2015; Accepted May 6, 2016

DOI: $10.3892 /$ etm.2016.3311

\begin{abstract}
Polyacrylamide hydrogel (PAHG) is a medical soft tissue filling agent utilized for the treatment of a variety of soft tissue defects. However, postoperative complications occur that have to be alleviated. The aim of the present study was to examine the treatments of postoperative complications of mammoplasty augmentation with PAHG. Open suction techniques and partial mastectomies via periareolar incisions were performed in 28 patients who had been injected with PAHG for breast augmentation. The PAHG was removed precluding breast complications resulting from the hydrogel injections. The symptoms disappeared completely, and the results were satisfactory following removal of the PAHG. In conclusion, the periareolar approach is valuable and removes PAHG to the greatest extent.
\end{abstract}

\section{Introduction}

Polyacrylamide hydrogel (PAHG) is a medical soft tissue filling agent employed for the treatment of a variety of soft tissue defects, since its introduction in the Ukraine in 1997 (?). PAHG has been used successfully in the plastic and reconstructive surgery of soft tissue defects. Augmentation mammoplasty with PAHG injection has also been used with success. However, there are postoperative complications and the increasing number of cases result in morbidity (?). The occurrence rate of such complications was reported to be $\leq 6-7 \%$ (?). Induration accounted for $58.89 \%$ of complications, with $10.11 \%$ being hematoma, $7.78 \%$ aseptic inflammation, $6 \%$ infection, $4.565 \%$ myositis, $3.44 \%$ asymmetric, $3.44 \%$ of PAHG leakage, $1.22 \%$ of displacement and $3 \%$ of other types of complications (1).

From January 2008 to October 2012, 28 patients with breast augmentation were successfully treated with PAHG injections.

Correspondence to: Dr Wang Wei, Department of Plastic Surgery, The First People's Hospital of Xuzhou, 19 Zhongshanbei Road, Xuzhou, Jiangsu 221000, P.R. China

E-mail:wv1210@126.com

Key words: polyacrylamide hydrogel, breast augmentation, complication
The aim of the present study was to examine the treatment of complications of breast augmentation with PAHG injections.

\section{Materials and methods}

Patients. From January 2008 to October 2012, 28 patients underwent breast augmentation with PAHG injections. The patients were 20-40 years of age, with an average age of 30.2 years. The post-injection time ranged from 2 months to 4 years, and the injection volume was approximately 150-200 $\mathrm{ml}$. However, the specific injection volume of the PAHG by individual case was not determined. The common complications were nodular breast lumps, chest pain, distorted breast and displacement of the PAHG. The majority of patients experienced several complications.

Methods. Incision on the subareolar or periareolar or subglandular plica was performed. After the skin and subcutaneous tissue incision, the superficial gland envelope was dissected until the lower pole of the gland. Separation in the deep part was then performed and the gland post gap was opened. When the PAHG was at appropriate levels, a granular, soft, jam-like substance was effused. In the case of several cavity gaps, an incision was performed in the membranes to form a connected cavity gap. The suction duct apparatus was subsequently inserted into the gap and filled with normal saline. During the lavage, the breast was massage to ensure PAHG effusion. When the gland or muscle was involved, an incision was made to the gland or myolemma and the PAHG was removed by curettage. In the case of the envelope being extremely thick or the sticky deformation of the tissue overly distinct, part of the muscle or gland was severed and sent for pathological examination. Normal saline was used to lavage the sections until the flushing fluid became translucent again. The gland or muscle tissue was probed mannually to ensure there were no nodules, and the breast was softer than prior to the procedure.

The present study was approved by the ethics committee of the First People's Hospital of Xuzhou (Jiangsu, China). Patient consent was obtained from the patient or the patient's family.

\section{Results}

General. After a 3 month- to 2-year follow-up, preoperative symptoms, such as sclerosis of the skin, breast nodule and pain had almost disappeared following surgery. For the patients 


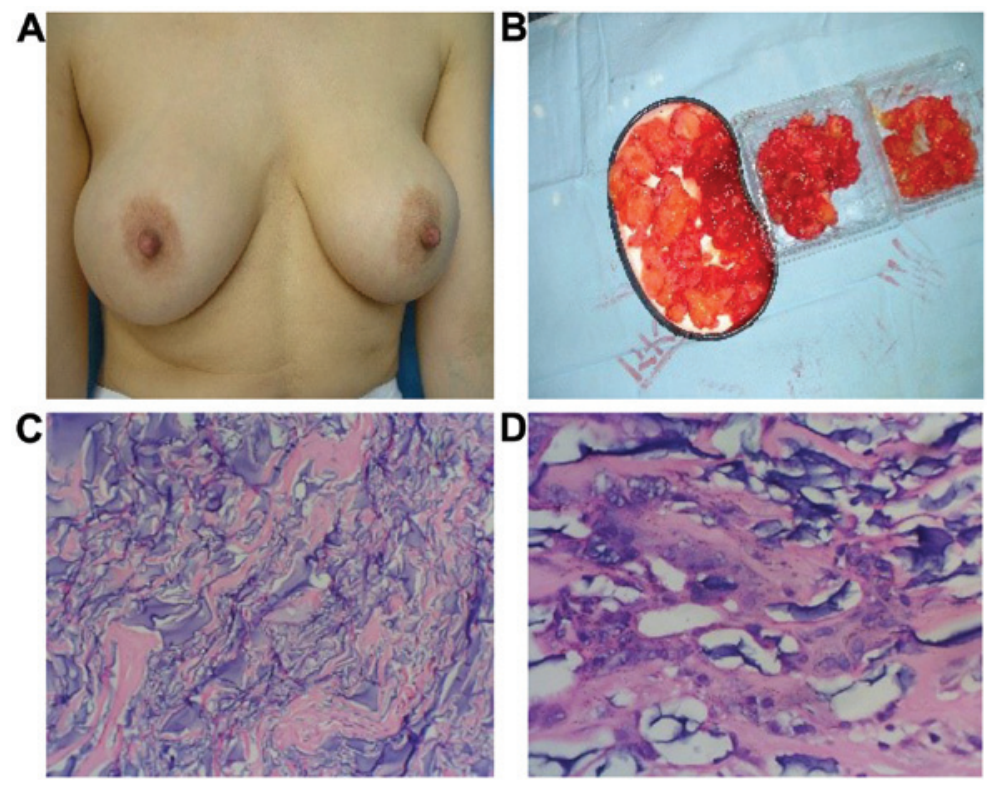

Figure 1. (A) Distorted breast 1 year after the augmentation mammoplasty by polyacrylamide hydrogel injection. (B) Granular, soft and jam-like injectants were removed during the operation. (C and D) The pathological examination shows the fibrous tissue were proliferating and masses of inflammatory mononuclear cells and macrophage were infiltrating into the tissue.

A
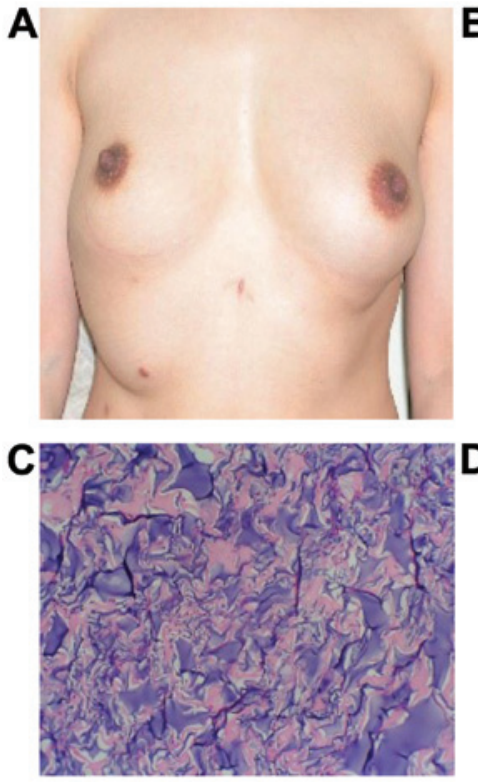

B

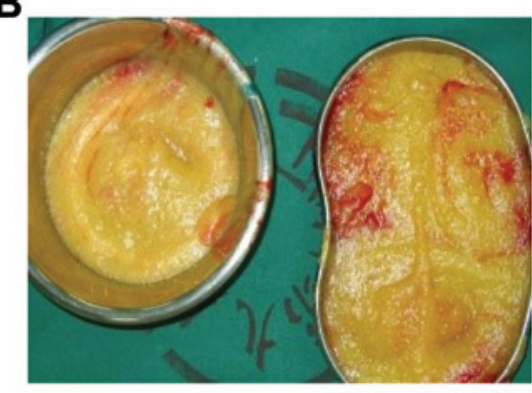

D

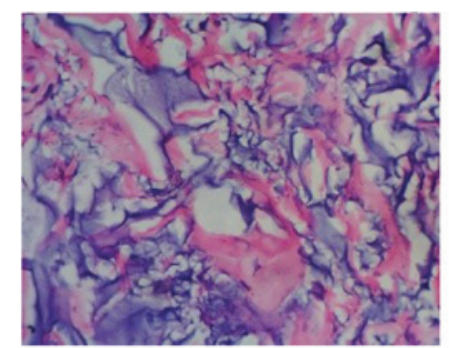

Figure 2. (A) The injectants were repositioned after 4 years of the operation and the patient seemed to have four breasts. (B) The jam-like hydrogel was removed. (C and D) The pathological examination shows that it comprised collagenous fiber and fibroblast and infiltrated with a great number of mononuclear cells.

who underwent breast augmentation prosthesis, the breasts were soft and bilaterally symmetric, with a good appearance. There were no symptoms of infection, pain or lumps in any of the patients. The patients expressed satisfaction with the postoperative effect.

Typical cases. Case 1 was a female patient, aged 36 years and married. She underwent PAHG injection because of light breast ptosis in another hospital. Although two months post-surgery the patient experienced chest pain, especially when she breathed, she did not pay particular attention to the symptom. After 1 year, the mammary gland was hardened, distorted and tender. She tried to communicate with the hospital but failed. After 2 years, the patient presented at the First People's Hospital of Xuzhou (Jiangsu, China), where she was diagnosed as having complications from the augmentation mammoplasty with the PAHG. Surgery was carried out. Incision over the areolar was performed, and we found that there were many tender nodules of various shapes and sizes in the glandular tissue, retromammary cellular space, post-pectoralis major space, and pectoralis major and minor muscles. The glands and muscles involved were edematous and distorted. We removed the granular hydrogel, and the distorted tissue. The pathological examination showed the fibrous tissues were 
proliferating, and masses of inflammatory mononuclear cells were being infiltrated by the tissue. The paient was advised to undergo prosthetic plombage surgery. Through a telephone follow-up, we learned that the symptoms disappeared, and the patient was satisfied with the results (Fig. 1).

Case 2 was a female patient, aged 32 years and married. She underwent PAHG injections in another hospital with relatively positive results and symmetrical breasts. Six months prior to presenting at the hospital, the patient underwent laparoscopy for cholecystitis. During surgery, it was found that there were lumps in her breasts, and the breasts were distorted. The medical history revealed chest pain subsequent to the surgery. Owing to the complications of augmentation mammoplasty by PAHG injection, the hydrogel was removed. Incision of plica under breast was performed, and the enclosed massed presented in the subcutaneous tissue and mammary glands in a number of various shapes and sizes. There were also incomplete and irregular envelopes in the multilocular cystic condition. An incision was made in the myolemma of the pectoralis major, and the PAHG that was distributed in the muscle bundles was drained and then flushed with normal saline. Part of the removed envelopes, which were sent for pathological examination, comprised collagenous fiber and fibroblast. The foreign body surface was infiltrated with a great number of mononuclear cells. At 2 weeks following surgery, the pain symptoms disappeared, the breasts were asymmetrical, and the breasts collapsed. The patient did not undergo any further breast augmentation (Fig. 2).

\section{Discussion}

Hydrophilic polyacrylamide hydrogel, or HPAMG, is broadly used as an injectant in breast augmentation due to its relatively good physiological compatibility and steady physicochemical property (2). However, the complications of surgery leads to the patient experiencing pain.

The filling material of the injection in breast augmentations should be placed in the retromammery cellular space (6). However, since the injection performed is obscured, the position into which the material is injected is often inaccurate. In addition, a large volume of the material, reaching 150-200 ml, is required $(7,8)$. Under the influence of gravity, body positions, muscular movement and postoperative massage, the injectant may infiltrate into the surrounding tissue and redistribute itself within the body. The injectants may distribute in the form of crumbs in the outer and downside of the submammary gland space, inside of the mammary gland or subcutaneous tissue, or even the outer side of the breasts $(7,8)$.

The most common complication, which is the formation of breast induration following breast augmentation by PAHG, has been identified in almost all the clinical cases (9). The breast induration varies in size and is distributed in subcutaneous tissue, glands or muscles. Although the hydrogel is regarded as a biomaterial that cannot cause cancer or malformation, the formation of lumps has obvious interference over the differential diagnosis of an early mammary tumor (10). Chest pain is also a common complication and can primarily be identified in the pectorals where the hydrogel is located. Previous findings suggest that hydrogel does not move subsequent to the injection (11). However, there is a part of the hydrogel that is transferred to the surrounding tissue over time, with some hydrogel being transferred beyond the scope of breasts and reaching the armpits or napes.

When selecting a method to remove the hydrogel, liposuction using the tumescent technique can be used for patients whose injection in the early period was only limited to retromammary cellular space (12). However, for patients with decentralized crumbs, or those whose injection is located in the pectoralis major, liposuction is not as easy. Injectants become a granulate and multicystic enclosed mass after a long period of time has elapsed (12). Additionally, the diolame is separated in the net structure (12). These issues make liposuction difficult to perform. In addition, this method can also damage the surrounding tissue, displace the hydrogel during the piercing process and develop several cavity spaces. Thus, incision on the subareolar or periareolar or subglandular plica is performed. The envelope of glands is stripped from the subcutaneous tissue and the shell is sectioned, resulting in the injectants emerging. At the same time, this method can remove the distorted muscle $(13,14)$.

Owing to the infiltration of hydrogel, we cannot ensure that the hydrogel is completely removed under direct vision. However, it plays a decisive role in symptom relief, relieves psychological burden and there is no interference of early screening on the mammary tumor (10). Only by complete removal of the subcutaneous glands and muscle can the hydrogel be thoroughly removed. Patients remain unsatisfied with the changes in the shape of the breasts. Breast augmentation prosthesis is therefore not performed unless the patients consent to the procedure. Six months post-surgery, a B ultrasonic super-review may be conducted. If no obvious remaining hydrogel is identified, the patient may consider accepting the silicone gel breast implant to improve the breast shape $(15,16)$.

In conclusion, the introduction of PAHGs in breast augmentation is a recent development and there are insufficient large samples of animal experiments and clinical trials to thoroughly determine its effects. Although PAHGs possess better histocompatibility, there are complications that should be considered (9). Thus, this method may not the optimal choice for breast augmentation.

\section{References}

1. Kasi AD, Pergialiotis V, Perrea DN, Khunda A and Doumouchtsis SK: Polyacrylamide hydrogel (Bulkamid(R)) for stress urinary incontinence in women: A systematic review of the literature. Int Urogynecol J 27: 367-375, 2016.

2. Feng XL, Yi CX, Peng C, Zhang YM, Yang M, Wang YR, Guo NQ and Zhou WD: Analysis of the complications induced by polyacrylamide hydrogel injection. Plast Reconstr Surg 114: 261-262, 2004.

3. Do ER and Shim JS: Long-term complications from breast augmentation by injected polyacrylamide hydrogel. Arch Plast Surg 39: 267-269, 2012.

4. Zhou Z, Liu DL, Lu KH, et al: The research of complications in syringe breast augmentation using polyacrylamide hydrogel. Chin J Pratic Aesthet Plast Surg 16: 353-356, 2005.

5. Wang HY, Jiang YX and Qiao Q: Ultrasonographic value for thecomplications of breast augmentation with injectable polyacrylamide hydrogel technique. Zhonghua Zheng Xing Wai Ke Za Zhi 23: 97-100, 2007 (In Chinese).

6. Biggs TM, Yarish RS: Augmentation mammaplasty: Retropectoral versus retromammary implantation. Clin Plast Surg 15: 549-555, 1988. 
7. Godwin Y, Duncan RT, Feig C, Reintals M and Hill S: Soft, Brown Rupture: Clinical Signs and Symptoms Associated with Ruptured PIP Breast Implants. Plast Reconstr Surg Glob Open 2: e249, 2014

8. Kim CH: Asian Outcomes of Primary Breast Augmentation in 162 Consecutive Cases by a Single Surgeon. Plast Reconstr Surg Glob Open 3: e537, 2015.

9. Cheng NX, Wang YL, Wang JH, Zhang XM and Zhong $\mathrm{H}$ : Complications of breast augmentation with injected hydrophilic polyacrylamide gel. Aesthetic Plast Surg 26: 375-382, 2002.

10. Liu HL and Cheung WY: Complications of polyacrylamide hydrogel (PAAG) injection in facial augmentation. J Plast Reconstr Aesthet Surg 63: e9-e12, 2010.

11. Chao MJ and Yin WM: Application of hydrophilic polyacrylamide gel in augmentation mammoplasty. J Pract Aesth Plast Surg 11: 16-18, 2000 (In Chinese).
12. Leung KM, Yeoh GP and Chan KW: Breast pathology in complications associated with polyacrylamide hydrogel (PAAG) mammoplasty. Hong Kong Med J 13: 137-140, 2007.

13. Wang Z, Li S, Wang L, Zhang S, Jiang Y, Chen J and Luo D: Polyacrylamide hydrogel injection for breast augmentation: Another injectable failure. Med Sci Monit 18: CR399-CR408, 2012.

14. Ahmed EM: Hydrogel: Preparation, characterization, and applications: A review. J Adv Res 6: 105-121, 2015.

15. Losken A: Early Results Using Sterilized Acellular Human Dermis (Neoform) in Post-Mastectomy Tissue Expander Breast Reconstruction. Plast Reconstr Surg 123: 1654-1658, 2009.

16. Manafi A, Emami AH, Pooli AH, Habibi M and Saidian L: Unacceptable results with an accepted soft tissue filler: Polyacrylamide hydrogel. Aesthetic Plast Surg 34: 413-422, 2010. 